\title{
Status and progress of the HFLAV-Tau group activities
}

\author{
Alberto Lusiani ${ }^{1,2, \star}$ \\ ${ }^{1}$ Scuola Normale Superiore, Pisa, Italy \\ ${ }^{2}$ INFN sezione di Pisa, Pisa, Italy
}

\begin{abstract}
We report the status and progress of the Heavy Flavour Averaging Group work on the global fit of $\tau$ tau lepton branching fractions, on the lepton universality tests and on the measurement of the Cabibbo-Kobayashi-Maskawa matrix element $\left|\boldsymbol{V}_{u s}\right|$ using $\tau$ lepton measurements. We also review the prospects for improving the precision of the $\left|V_{u s}\right|$ measurement.
\end{abstract}

\section{Introduction}

To best exploit the many experimental measurements on the $\tau$ lepton branching fractions, it is convenient to perform a global fit. The results are useful to compute the lepton universality tests, the strong coupling constant at the $\tau$ mass, the Cabibbo-Kobayashi-Maskawa (CKM) matrix element $\left|V_{u s}\right|$ and the muon $g-2$ hadronic vacuum polarization contribution.

The $\tau$ subgroup of the Heavy Flavour Averaging Group (HFLAV) has updated in 2016 the global fit of the $\tau$ branching fractions, the lepton universality tests and the $\left|V_{u s}\right|$ determination based on $\tau$ measurements. A version of the global fit with unitarity constraint has been published in the Review of Particle Physics [1] (RPP). All the 2016 HFLAV-Tau results have been submitted for publication as part of the 2016 HFLAV report [2]. These results are labelled "Spring 2017" and have only minimal differences to formerly reported preliminary results [3].

In the following, we summarize the latest HFLAV-Tau results, briefly mentioning the changes since the HFLAV 2014 report [4] and the minor modifications since the Tau 2016 conference update [3]. Furthermore, we review the prospects for improving the $\left|V_{u s}\right|$ determination based on $\tau$ measurements.

\section{Branching fractions fits}

A global fit of the available experimental measurements is used to determine the $\tau$ branching fractions, together with their uncertainties and statistical correlations. According to the HFLAV methodology [5], we considered all significant correlations induced by common systematic effects across different measurements, and we updated the measurements to take into account updates on other Physics quantities from which each specific measurement has documented dependencies. After doing that, we get a statistically consistent global fit by applying just a single scale factor of 5.44 to the

\footnotetext{
^e-mail: alberto.lusiani@pi.infn.it
} 
published uncertainties of the measurements of $\Gamma_{96}=\tau \rightarrow K K K v$ by BABAR and Belle, which would be otherwise severely statistically inconsistent.

The HFLAV Spring $2017 \tau$ branching fractions fit has $\chi^{2} /$ d.o.f. $=137 / 123$, corresponding to a confidence level $\mathrm{CL}=17.84 \%$. The procedure uses a total of 170 measurements to fit 135 quantities subjected to 88 constraints. The fit is statistically consistent with unitarity, and the unitarity residual is $1-\Gamma_{\text {All }}=(0.0355 \pm 0.1031) \cdot 10^{-2}$.

With respect to the $2014 \mathrm{HFLAV}$ report, we removed the Belle result on $\tau^{-} \rightarrow K_{S}^{0}$ (particles) ${ }^{-} v_{\tau}$ [6] because the published information does not permit a reliable determination of the correlations with the other results in the same paper. The inclusion of that result made the correlation matrix of the 2014 fit results non positive-definite. Furthermore, as detailed elsewhere [2, 3], we discarded some old preliminary results that are not expected to be published, we removed a result that was effectively $100 \%$ correlated with other measurements by the same experiment, and applied a few minor corrections to the constraints that relate different branching fractions. We have found that all these modifications have negligible impact on the lepton universality and the $\left|V_{u s}\right|$ measurements.

As is standard for the RPP branching fraction fits, the RPP $2016 \tau$ branching fraction fit provided by HFLAV-Tau is unitarity constrained, while the HFLAV Spring 2017 fit is not. In the RPP 2016, the ALEPH experiment measurements are used in a slightly different way compared to the HFLAV fit, as documented elsewhere [2, 3]. The two fits also have a slightly different treatment of the experimental results related to $\Gamma_{805}=B\left(\tau \rightarrow a_{1}^{-}\left(\rightarrow \pi^{-} \gamma\right) \nu_{\tau}\right)$ [2, 3].

\section{Tests of lepton universality}

According to the Standard Model (SM), the partial width of a heavier lepton $\lambda$ decaying to a lighter lepton $\rho$ [7] is,

$$
\Gamma\left(\lambda \rightarrow v_{\lambda} \rho \bar{v}_{\rho}(\gamma)\right)=\frac{B\left(\lambda \rightarrow v_{\lambda} \rho \bar{v}_{\rho}\right)}{\tau_{\lambda}}=\frac{G_{\lambda} G_{\rho} m_{\lambda}^{5}}{192 \pi^{3}} f\left(\frac{m_{\rho}^{2}}{m_{\lambda}^{2}}\right) R_{W}^{\lambda} R_{\gamma}^{\lambda},
$$

where

$$
\begin{aligned}
G_{\rho} & =\frac{g_{\rho}^{2}}{4 \sqrt{2} M_{W}^{2}}, & f(x) & =1-8 x+8 x^{3}-x^{4}-12 x^{2} \ln x, \\
R_{W}^{\lambda} & =1+\frac{3}{5} \frac{m_{\lambda}^{2}}{M_{W}^{2}}+\frac{9}{5} \frac{m_{\rho}^{2}}{M_{W}^{2}}, & R_{\gamma}^{\lambda} & =1+\frac{\alpha\left(m_{\lambda}\right)}{2 \pi}\left(\frac{25}{4}-\pi^{2}\right) .
\end{aligned}
$$

With respect to the recently reported preliminary results [3], we include now the subleading term to $R_{W}^{\lambda}$ [8, 9], which does not change the reported numbers. We use $R_{\gamma}^{\tau}=1-43.2 \cdot 10^{-4}$ and $R_{\gamma}^{\mu}=$ $1-42.4 \cdot 10^{-4}$ [7] and $M_{W}$ from PDG 2015 [10]. We use HFLAV Spring 2017 averages and PDG 2015 for the other quantities. Using leptonic processes we obtain

$$
\left(g_{\tau} / g_{\mu}\right)=1.0010 \pm 0.0015, \quad\left(g_{\tau} / g_{e}\right)=1.0029 \pm 0.0015, \quad\left(g_{\mu} / g_{e}\right)=1.0019 \pm 0.0014
$$

Figure 1 shows the test of the SM prediction of the relation between the $\tau$ leptonic branching fractions $B_{\ell}=B\left(\tau \rightarrow \ell \bar{v}_{\ell} \nu_{\tau}\right)$, with $\ell=e, \mu$, the $\tau$ lifetime $\tau_{\tau}$, the $\tau$ mass $m_{\tau}$, and the respective muon parameters,

$$
B_{\tau \ell}^{\mathrm{SM}}=B_{\mu e} \frac{\tau_{\tau}}{\tau_{\mu}} \frac{m_{\tau}^{5}}{m_{\mu}^{5}} \frac{f_{\tau \ell} r_{W}^{\tau} r_{\gamma}^{\tau}}{f_{\mu e} r_{W}^{\mu} r_{\gamma}^{\mu}}, \quad \text { with } f_{\lambda \rho}=f\left(\frac{m_{\rho}^{2}}{m_{\lambda}^{2}}\right) .
$$




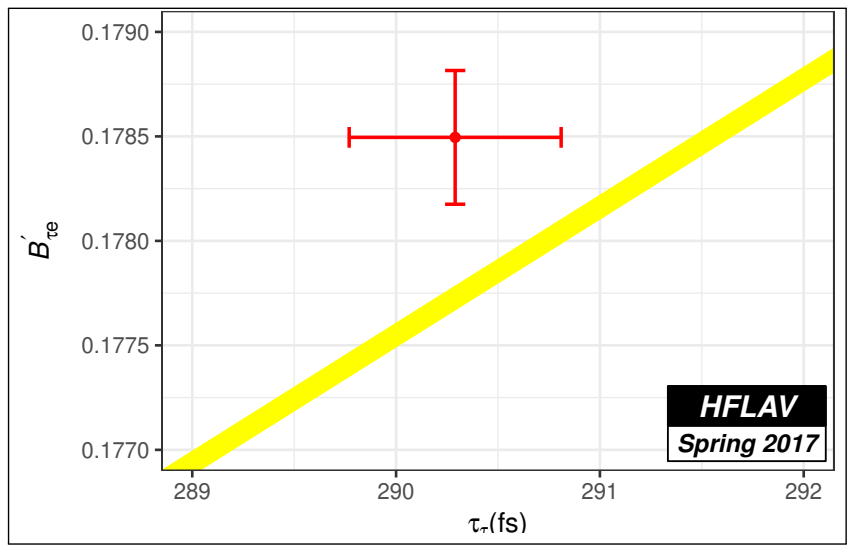

Figure 1. Test of the SM prediction of the relation between the $\tau$ leptonic branching fractions and the $\tau$ lifetime and mass. $B_{\tau e}^{\prime}$ denotes the statistical average of $B_{e}=B\left(\tau \rightarrow e \bar{v}_{e} v_{\tau}\right)$ and the $B_{e} \mathrm{SM}$ prediction from the $B_{\mu}$ measurement $B_{e}\left(B_{\mu}\right)=B_{\mu} \cdot\left(f_{\tau e} / f_{\tau \mu}\right)$. The yellow band represents the uncertainty from the $\tau$ lifetime.

Using semi-hadronic processes

$$
\left(\frac{g_{\tau}}{g_{\mu}}\right)^{2}=\frac{B\left(\tau \rightarrow h v_{\tau}\right)}{B\left(h \rightarrow \mu \bar{v}_{\mu}\right)} \frac{2 m_{h} m_{\mu}^{2} \tau_{h}}{\left(1+\delta_{h}\right) m_{\tau}^{3} \tau_{\tau}}\left(\frac{1-m_{\mu}^{2} / m_{h}^{2}}{1-m_{h}^{2} / m_{\tau}^{2}}\right)^{2},
$$

where $h=\pi$ or $K$ and the radiative corrections are $\delta_{\pi}=(0.16 \pm 0.14) \%$ and $\delta_{K}=(0.90 \pm 0.22) \%$ [11]. We measure:

$$
\left(g_{\tau} / g_{\mu}\right)_{\pi}=0.9961 \pm 0.0027, \quad\left(g_{\tau} / g_{\mu}\right)_{K}=0.9860 \pm 0.0070
$$

Similar tests could be performed with decays to electrons, however they are less precise because the hadron two body decays to electrons are helicity-suppressed. Averaging the three $g_{\tau} / g_{\mu}$ ratios we obtain

$$
\left(g_{\tau} / g_{\mu}\right)_{\tau+\pi+K}=1.0000 \pm 0.0014
$$

accounting for all correlations.

\section{$4\left|V_{u s}\right|$ measurement}

The measurements of the kaon branching fractions are used in conjunction with lattice QCD estimates of hadronic form factors to provide the most precise determinations of $\left|V_{u s}\right|$ [1]. The $\tau$ exclusive branching fractions to strange final states can be used in a similar way to obtain additional less precise $\left|V_{u s}\right|$ determinations. Furthermore, the inclusive branching fraction of the $\tau$ to all strange final states, $B\left(\tau \rightarrow X_{s} v\right)$, can be used to compute $\left|V_{u s}\right|$ with a procedure that does not require lattice QCD estimates and has an independent and small theory uncertainty [12]:

$$
\left|V_{u s}\right|_{\tau s}=\sqrt{R_{s} /\left[\frac{R_{\mathrm{VA}}}{\left|V_{u d}\right|^{2}}-\delta R_{\text {theory }}\right]} .
$$

$R_{s}$ and $R_{\mathrm{VA}}$ are the $\tau$ hadronic partial widths to strange and to non-strange hadronic final states $\left(\Gamma_{s}\right.$ and $\left.\Gamma_{\text {had }}\right)$ divided by the universality-improved branching fraction $B(\tau \rightarrow e v \bar{v})=B_{e}^{\text {uni }}=$ $(17.815 \pm 0.023) \%$ [2, 3]. We compute $\delta R_{\text {theory }}=0.242 \pm 0.032$ using inputs from Ref. [12] 


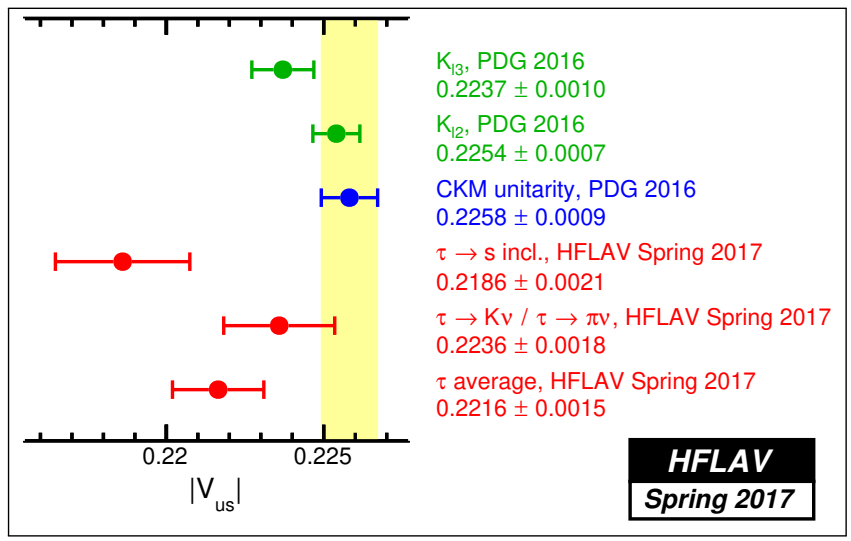

Figure 2. $\left|V_{u s}\right|$ computed using the $\tau$ measurements compared to the determinations based on the CKM matrix unitarity and on the kaon branching fractions.

and $m_{s}=(95.00 \pm 5.00) \mathrm{MeV}[10]$. Alternative determinations exist [12]-14]. We use $\left|V_{u d}\right|=$ $0.97417 \pm 0.00021$ [15]. We obtain $\left|V_{u s}\right|_{\tau s}=0.2186 \pm 0.0021$, which is $3.1 \sigma$ lower than the unitarity CKM prediction $\left|V_{u s}\right|_{\text {uni }}=0.22582 \pm 0.00089$, from $\left(\left|V_{u s}\right|_{\text {uni }}\right)^{2}=1-\left|V_{u d}\right|^{2}\left(\left|V_{u b}\right|\right.$ is negligible $)$. The $\left|V_{u s}\right|_{\tau s}$ uncertainty includes a systematic uncertainty contribution of $0.47 \%$ from the theory uncertainty on $\delta R_{\text {theory }}$.

We follow the same procedure of the HFLAV 2012 report [16] to compute $\left|V_{u s}\right|$ from the ratio of branching fractions $B\left(\tau \rightarrow K^{-} v_{\tau}\right) / B\left(\tau \rightarrow \pi^{-} v_{\tau}\right)=(6.438 \pm 0.094) \cdot 10^{-2}$ from the equation

$$
\frac{B\left(\tau \rightarrow K^{-} v_{\tau}\right)}{B\left(\tau \rightarrow \pi^{-} v_{\tau}\right)}=\frac{f_{K}^{2}\left|V_{u s}\right|^{2}}{f_{\pi}^{2}\left|V_{u d}\right|^{2}} \frac{\left(1-m_{K}^{2} / m_{\tau}^{2}\right)^{2}}{\left(1-m_{\pi}^{2} / m_{\tau}^{2}\right)^{2}} R_{\tau K / \tau \pi} .
$$

We use $f_{K} / f_{\pi}=1.1930 \pm 0.0030$ from the FLAG 2016 Lattice averages with $N_{f}=2+1+1$ [17]. The HFLAV Spring 2017 report [2] contains more details on the radiative correction term $R_{\tau K / \tau \pi}=$ $0.9930 \pm 0.0035$. We obtain $\left|V_{u s}\right|_{\tau K / \pi}=0.2236 \pm 0.0018,1.1 \sigma$ below the CKM unitarity prediction.

Until the most recent HFLAV-Tau update [3], we reported a third $\left|V_{u s}\right|$ measurement using $B(\tau \rightarrow$ $K v)$. The HFLAV Spring 2017 update does not include any more that last determination, because it did not include the long-distance radiative corrections in addition to the short-distance contribution. This change has a negligible effect on the overall precision of $\left|V_{u s}\right|$.

Averaging the two $\left|V_{u s}\right|$ determinations, we obtain $\left|V_{u s}\right|_{\tau}=0.2216 \pm 0.0015$, which is $2.4 \sigma$ below the CKM unitarity determination. Figure 2 summarizes the $\left|V_{u s}\right|$ results, reporting also $\left|V_{u s}\right|$ computed from kaon measurements [1] and $\left|V_{u s}\right|$ obtained from $\left|V_{u d}\right|$ and the CKM matrix unitarity [1].

\section{Studies on $\left|V_{u s}\right|$ from $\tau$ data}

Assuming the SM, the following $\tau$ branching fractions have been computed using the precisely measured $K_{\ell 2}$ and $K_{\ell 3}$ branching fractions and the measured $\tau^{-} \rightarrow(K \pi)^{-} \nu_{\tau}$ spectra [18]:

$$
\begin{array}{ll}
B\left(\tau^{-} \rightarrow K^{-} v_{\tau}\right) & =(0.713 \pm 0.003) \%, \\
B\left(\tau^{-} \rightarrow K^{-} \pi^{0} v_{\tau}\right) & =(0.471 \pm 0.018) \%, \\
B\left(\tau^{-} \rightarrow K^{0} \pi^{-} v_{\tau}\right) & =(0.857 \pm 0.030) \%
\end{array}
$$

It has been observed [18, 19] that all the above indirect values are higher than the corresponding directly measured $\tau$ branching fractions. If the indirect values replace the direct ones, 
$\left|V_{u s}\right|=0.2207 \pm 0.027[18]$. If the indirect values are instead statistically combined with the direct measurements used in the HFLAV Spring 2017 global fit according to their respective uncertainties, we obtain $\left|V_{u s}\right|=0.2195 \pm 0.021$. Both $\left|V_{u s}\right|$ values are close to the one computed using just the HFLAV Spring 2017 fit outputs.

An alternative determination of $\left|V_{u s}\right|=0.2231 \pm 0.0027$ (stat.) \pm 0.0004 (syst.) [20] relies on the $\tau$ spectral functions in addition to the inclusive $\tau \rightarrow X_{s} v$ branching fraction, for the purpose of reducing the QCD-related uncertainties. This study replaces the HFLAV fit values for $B\left(\tau^{-} \rightarrow K^{-} v_{\tau}\right), B\left(\tau^{-} \rightarrow\right.$ $\left.K^{-} \pi^{0} v_{\tau}\right)$ and $B\left(\tau^{-} \rightarrow K^{0} \pi^{-} v_{\tau}\right)$ with the indirectly values in Ref. [18]. This last $\left|V_{u s}\right|$ value is entirely consistent with CKM unitarity.

A PHD thesis published in 2011 [21] reports measurements of $4 \tau$ branching fractions with a kaon in the finel state, performed in a not-yet-released BABAR analysis:

$$
\begin{array}{ll}
B(\tau \rightarrow K v) & =(0.710 \pm 0.003 \text { (stat.) } \pm 0.016 \text { (syst. })) \% \\
B\left(\tau \rightarrow K \pi^{0} v\right) & =(0.500 \pm 0.002 \text { (stat.) } \pm 0.014 \text { (syst. })) \\
B\left(\tau \rightarrow K 2 \pi^{0} v\right) & =(5.66 \pm 0.14 \text { (stat. } \pm 0.32 \text { (syst. })) \cdot 10^{-4} \\
B\left(\tau \rightarrow K 3 \pi^{0} v\right) & =(1.64 \pm 0.28 \text { (stat.) } \pm 0.38 \text { (syst.) }) \cdot 10^{-4}
\end{array}
$$

The 3 measurements with neutral pions in the final state correspond to 3 out of the 5 largest contributions to the uncertainty on $\left|V_{u s}\right|$ from the $\tau$ branching fractions. If we add to the HFLAV Spring 2017 inputs the above 4 results and the additional measurements from the same reference on $B\left(\tau \rightarrow \pi 3 \pi^{0} v\right)$ and $B\left(\tau \rightarrow \pi 4 \pi^{0} v\right)$, we perform the global $\tau$ branching fraction fit and we use the fit results, we obtain $\left|V_{u s}\right|=0.2192 \pm 0.019$, close to the HFLAV Spring 2017 value.

The main contributions to the HFLAV-Tau $\left|V_{u s}\right|$ determination uncertainty come from the $\tau$ branching fractions to strange final states and from the theory. After we add to the HFLAV-Tau $\tau$ measurements both the above mentioned in-progress $B A B A R$ results and the indirect measurements from the kaon branching fractions, the following list shows the resulting individual contributions to the $\left|V_{u s}\right|$ uncertainty.

$\begin{array}{ll}\left.\pi^{-} \bar{K}^{0} \pi^{0} \pi^{0} v_{\tau} \text { (ex. } K^{0}\right) & 0.3908 \\ \bar{K}^{0} h^{-} h^{-} h^{+} v_{\tau} & 0.3430 \\ K^{-} \pi^{-} \pi^{+} \pi^{0} v_{\tau}\left(\text { ex. } K^{0}, \omega, \eta\right) & 0.2422 \\ \pi^{-} \bar{K}^{0} \pi^{0} v_{\tau} & 0.2178 \\ K^{-} \omega v_{\tau} & 0.1563 \\ \pi^{-} \bar{K}^{0} v_{\tau} & 0.1501 \\ \left.K^{-} \pi^{-} \pi^{+} v_{\tau} \text { (ex. } K^{0}, \omega\right) & 0.1140 \\ K^{-} \pi^{0} v_{\tau} & 0.0881 \\ \left.K^{-} 3 \pi^{0} v_{\tau} \text { (ex. } K^{0}, \eta\right) & 0.0703 \\ K^{-} v_{\tau} & 0.0473 \\ K^{-} 2 \pi^{0} v_{\tau}\left(\text { ex. } K^{0}\right) & 0.0414 \\ \pi^{-} \bar{K}^{0} \eta v_{\tau} & 0.0253 \\ K^{-} \pi^{0} \eta v_{\tau} & 0.0197 \\ K^{-} \eta v_{\tau} & 0.0136 \\ K^{-} \phi v_{\tau}\left(\phi \rightarrow K^{+} K^{-}\right) & 0.0136 \\ K^{-} \phi v_{\tau}\left(\phi \rightarrow K_{S}^{0} K_{L}^{0}\right) & 0.0095 \\ K^{-} 2 \pi^{-} 2 \pi^{+} v_{\tau}\left(\text { ex. } K^{0}\right) & 0.0020 \\ K^{-} 2 \pi^{-} 2 \pi^{+} \pi^{0} v_{\tau}\left(\text { ex. } K^{0}\right) & 0.0010 \\ \tau \rightarrow \text { non-strange } & 0.0853 \\ \text { theory } & 0.4724\end{array}$

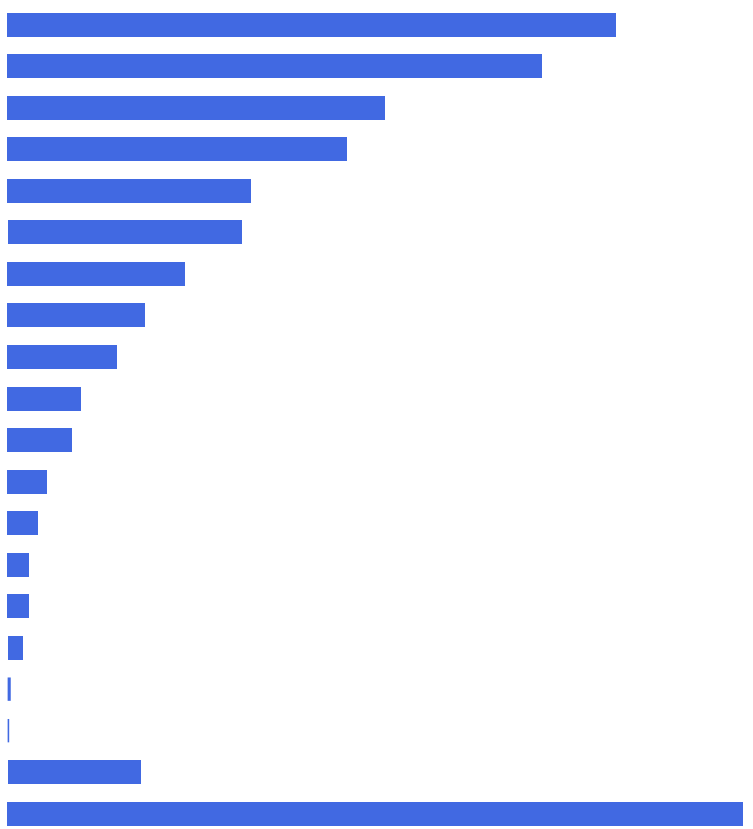


The Belle II super flavour factory may provide the required more precise measurements of the small $\tau$ strange branching fractions that presently limit the $\left|V_{u s}\right|$ precision. Furthermore, precision $\tau$ branching fractions and spectral function measurements can contribute to improving the theory uncertainty.

\section{Conclusions}

The Standard Model lepton universality is confirmed by the updated HFLAV Spring 2017 tests. The $\left|V_{u s}\right|$ determination from inclusive $\tau \rightarrow X_{s} v$ decays is $3.1 \sigma$ lower than the unitarity CKM prediction. The discrepancy persists also when considering indirect determinations of some $\tau$ branching fractions from kaon measurements and additional experimental measurements from an in-progress $B A B A R$ study. However, an alternative $\left|V_{u s}\right|$ computation relying also on the $\tau$ spectral functions obtains a $\left|V_{u s}\right|$ value that is entirely consistent with CKM unitarity. More precise $\tau$ branching fractions and spectral functions measurements are desirable to improve our understanding of the observed discrepancy on $\left|V_{u s}\right|$ from inclusive $\tau \rightarrow X_{s} v$ decays.

\section{References}

[1] C. Patrignani et al. (Particle Data Group), Chin. Phys. C40, 100001 (2016)

[2] Y. Amhis et al. (HFLAV), Eur. Phys. J. C77, 895 (2017), 1612.07233

[3] A. Lusiani, Nucl. Part. Phys. Proc. 287-288, 29 (2017)

[4] Y. Amhis et al. (Heavy Flavor Averaging Group (HFAG)) (2014), 1412.7515

[5] D. Asner et al. (Heavy Flavor Averaging Group), Averages of b-hadron, c-hadron, and $\tau$-lepton Properties (2010), 1010.1589

[6] S. Ryu et al. (Belle), Phys. Rev. D89, 072009 (2014), 1402 . 5213

[7] W. Marciano, A. Sirlin, Phys. Rev. Lett. 61, 1815 (1988)

[8] A. Ferroglia, C. Greub, A. Sirlin, Z. Zhang, Phys. Rev. D88, 033012 (2013), 1307.6900

[9] M. Fael, L. Mercolli, M. Passera, Phys. Rev. D88, 093011 (2013), 1310. 1081

[10] K. Olive et al., Chin. Phys. C38, 090001 (2014)

[11] R. Decker, M. Finkemeier, Phys. Lett. B334, 199 (1994)

[12] E. Gamiz, M. Jamin, A. Pich, J. Prades, F. Schwab, Nucl. Phys. Proc. Suppl. 169, 85 (2007), hep-ph/0612154

[13] E. Gamiz, M. Jamin, A. Pich, J. Prades, F. Schwab, PoS KAON, 008 (2008), 0709.0282

[14] K. Maltman, Nucl. Phys. Proc. Suppl. 218, 146 (2011), 1011.6391

[15] J.C. Hardy, I.S. Towner, Phys. Rev. C91, 025501 (2015), 1411.5987

[16] Y. Amhis et al. (Heavy Flavor Averaging Group) (2012), 1207. 1158

[17] S. Aoki et al., Eur. Phys. J. C77, 112 (2017), see also http://itpwiki.unibe.ch/flag/. 1607.00299

[18] M. Antonelli, V. Cirigliano, A. Lusiani, E. Passemar, JHEP 10, 070 (2013), 1304.8134

[19] A. Pich, Prog. Part. Nucl. Phys. 75, 41 (2014), 1310. 7922

[20] R.J. Hudspith, R. Lewis, K. Maltman, J. Zanotti, Phys. Lett. B781, 206 (2018), 1702 . 01767

[21] A. Adametz, Ph.D. thesis, Heidelberg U. (2011), http://www.ub.uni-heidelberg.de/ archiv/12325 